\title{
Treatment of Human Thyroid Carcinoma Cells with the G47delta Oncolytic Herpes Simplex Virus
}

\author{
Jia-Ni Wang ${ }^{1 \&}$, Li-Hua $\mathrm{Xu}^{2 \&}$, Wei-Gen Zeng ${ }^{1,3}$, Pan $\mathrm{Hu}^{1}$, Samuel D Rabkin ${ }^{4}$, \\ Ren-Rin Liu ${ }^{1 *}$
}

\begin{abstract}
Background: Thyroid carcinoma is the most common malignancy of the endocrine organs. Although the majority of thyroid cancer patients experience positive outcomes, anaplastic thyroid carcinoma is considered one of the most aggressive malignancies. Current therapeutic regimens do not confer a significant survival benefit, and new therapies are urgently needed. Oncolytic herpes simplex virus (oHSV) may represent a promising therapy for cancer. In the present study, we investigated the therapeutic effects of a third-generation HSV vector, G47A, on various human thyroid carcinoma cell lines in vitro. Two subcutaneous (s.c.) models of anaplastic thyroid carcinoma were also established to evaluate the in vivo anti-tumor efficacy of G47 $\Delta$. Materials and Methods: The human thyroid carcinoma cell line ARO, FRO, WRO, and KAT-5, were infected with G47 $\Delta$ at different multiplicities of infection (MOIs) in vitro. The survival rates of infected cells were calculated each day. Two s.c. tumor models were established using ARO and FRO cells in Balb/c nude mice, which were intratumorally (i.t.) treated with either G47 $\Delta$ or mock. Tumor volumes and mouse survival times were documented. Results: G47 $\Delta$ was highly cytotoxic to different types of thyroid carcinomas. For ARO, FRO, and KAT-5, greater than 30\% and $80 \%$ of cells were killed at MOI $=0.01$ and MOI=0.1, respectively on day 5 . WRO cells displayed modest sensitivity to G47 $\Delta$, with only $21 \%$ and $38 \%$ of cells killed. In the s.c. tumor model, both of the anaplastic thyroid carcinoma cell lines (ARO and FRO) were highly sensitive to G47 $\Delta$; G47 $\Delta$ significantly inhibited tumor growth and prolonged the survival of mice bearing s.c. ARO and FRO tumors. Conclusions: The oHSV G47 $\Delta$ can effectively kill different types of human thyroid carcinomas in vitro. G47 $\Delta$ significantly inhibited growth of anaplastic thyroid carcinoma in vivo and prolonged animal survival. Therefore, G47 $\Delta$ may hold great promise for thyroid cancer patients.
\end{abstract}

Keywords: Thyroid carcinoma - oncolytic herpes simplex virus - cytotoxicity - subcutaneous models

Asian Pac J Cancer Prev, 16 (3), 1241-1245

\section{Introduction}

Thyroid carcinoma, which consists of a group of diseases with different clinicopathologic features, is the most common malignancy of the endocrine organs (Vanderpump, 2011). Differentiated thyroid carcinoma (DTC) includes 2 types of malignancies: papillary thyroid carcinoma (PTC) and follicular thyroid carcinoma (FTC) (Zaman et al., 2012). DTC is the most common thyroid malignancy and is often associated with an indolent clinical course and 5-year survival rates greater than $90 \%$ (Sipos and Mazzaferri, 2010). However, no treatment has been proven to be effective for radioiodine-resistant metastatic disease, and the 10-year survival rate is less than $15 \%$ for these patients (Sherman et al., 2008). Medullary thyroid carcinoma (MTC) originates from the canceration of parafollicular C cells and presents a more aggressive clinical course than DTC. In addition, MTC is resistant to radioactive iodine therapy (Pacini et al., 2010; Vanderpump, 2011). Anaplastic thyroid carcinoma (ATC), or undifferentiated thyroid carcinoma, accounts for approximately 1-2\% of thyroid malignancies (Lin et al., 2008; Smallridge et al., 2012). ATC is one of the most aggressive tumor types, with a median survival time of no more than 6 months (Are and Shaha, 2006; Lin et al., 2008; Smallridge et al., 2012). In addition, one-half of all ATC patients have distant metastases at diagnosis, and radiation and chemotherapy are of little sensitivity and do not result in improvements in survival (Are and Shaha, 2006). Therefore, new therapies are needed to target thyroid tumors, especially metastases and ATC.

Oncolytic viruses selectively infect tumor cells and

${ }^{1}$ Breast Cancer Center, The Third Affiliated Hospital of Sun Yat-sen University, ${ }^{2}$ Department of Oncology and Hematology, The First Affiliated Hospital of Guangzhou Medical University, Guangzhou, ${ }^{3}$ Department of Colorectal Surgery, Cancer Hospital, Chinese Academy of Medical Sciences, Peking Union Medical College, Beijing, China, ${ }^{4}$ Department of Neurosurgery, Massachusetts General Hospital and Harvard Medical School, USA \&Equal contributors *For correspondence: liur@vip.163.com 
replicate within them. The tumor cells are then destroyed, and the viruses are released from them, infecting other tumor cells (Russell et al., 2012). Herpes simplex virus is one of the most common viruses used for tumor therapy. Oncolytic herpes simplex virus (oHSV) can be genetically modified, thereby increasing tumor selectivity and safety (Varghese and Rabkin, 2002). Preclinical and clinical experiments have demonstrated that oHSV can effectively and safely treat various tumors (Varghese and Rabkin, 2002). G47 $\Delta$ is a third-generation oHSV vector, and our previous studies have indicated that $\mathrm{G} 47 \Delta$ can effectively treat breast and nasopharyngeal carcinomas (Wang et al., 2011a; Wang et al., 2011b; Li et al., 2012; Zeng et al., 2013a; Zeng et al., 2013b).

In the present study, we investigated the cytotoxic effects of the G47D oHSV vector on various human thyroid cancer cell lines in vitro. Furthermore, we evaluated the therapeutic effects of G47 $\Delta$ on subcutaneous (s.c.) xenograft models of 2 human ATC cell lines.

\section{Materials and Methods}

\section{Ethics statement}

The animal work in the present study was conducted under the institutional guidelines of Guangdong Province and was approved by the Use Committee for Animal Care and the Sun Yat-sen University Institute Research Ethics Committee.

\section{Cell lines and virus}

The human papillary thyroid carcinoma cell line KAT-5, the human follicular thyroid carcinoma cell line WRO, and human undifferentiated thyroid carcinoma cell lines, ARO and FRO, were obtained from the European Collection of Cell Cultures (ECACC, Salisbury, United Kingdom). The cell lines were cultured in Roswell Park Memorial Institute (RPMI) 1640 Media containing glucose (4.5 g/l; Mediatech, Inc., Herndon, VA) and supplemented with $10 \%$ fetal calf serum (Hyclone Laboratories, Logan, $\mathrm{UT}$ ) and were grown at $37^{\circ} \mathrm{C}$ in $5 \% \mathrm{CO}_{2}$. Vero cells (African green monkey kidney cells purchased from The Committee on Type Culture Collection of the Chinese Academy of Sciences, Shanghai, China) were cultured in Dulbecco's Modified Eagle's Medium (DMEM) containing glucose (4.5 g/l; Mediatech, Inc., Herndon, VA) and supplemented with $10 \%$ fetal calf serum (Hyclone Laboratories, Logan, UT) and were grown at $37^{\circ} \mathrm{C}$ in $5 \%$ $\mathrm{CO}_{2}$. The oHSV G47 $\Delta$ was provided by MediGene, Inc. (San Diego, CA). G47 $\Delta$ is a conditionally replicating HSV-1 vector that was constructed as previously described (Todo et al., 2001). Briefly, the a47 gene, the promoter region of the US11 gene, and both copies of neurovirulence gene $\gamma 34.5$ were deleted, and the ICP6 gene was inactivated by the insertion of a lacZ gene. As a result of these genetic mutations, G47 $\Delta$ selectively replicates in tumor cells but spares normal cells.

\section{In vitro cytotoxicity}

The cancer cells were seeded in 6 well plates at densities of $1 \times 10^{5}$ cells per well. After 1 night of incubation, the cells were infected with the mock virus or G47D at multiplicities of infection (MOI) of 0.01 and 0.1. The cells were then cultured in RPMI 1640 media supplemented with $1 \%$ fetal calf serum (Hyclone Laboratories, Logan, UT) and grown at $37^{\circ} \mathrm{C}$ in $5 \% \mathrm{CO}_{2}$. The number of surviving cells was calculated each day using a hemocytometer. The average number of cells from triplicate wells are plotted as percentages of the mock wells. $\mathrm{X}$-gal histochemistry was also performed each day postinfection according to the manufacturer's recommended protocol (Beyotime Institute of Biotechnology, China). The infected cells expressed Lac Z and were stained blue.

\section{Animal studies}

Four-week-old female Balb/c nude mice were purchased from the Shanghai Institutes for Biological Sciences, CAS, Shanghai, China. Human undifferentiated thyroid carcinoma cells, ARO $\left(3 \times 10^{6}\right)$ and FRO $\left(3 \times 10^{6}\right)$, were suspended in $0.1 \mathrm{~mL}$ of RPMI 1640 media with $25 \%$ Matrigel (BD Biosciences) and implanted s.c. into the left flanks of 4-week-old nude mice. When the maximal diameter of the tumors reached approximately $5 \mathrm{~mm}$, the mice were randomly assigned to 1 of 2 groups $(6$ mice/ group): intratumoral (i.t.) injection of $50 \mu \mathrm{l}$ of virus buffer $[150 \mathrm{mM} \mathrm{NaCl}$ and $20 \mathrm{mM}$ Tris, $(\mathrm{pH} 7.5)]$ or $2 \times 10^{7}$ plaque forming units (pfu) of G47 $\Delta$ twice per week for 2 weeks. The lengths (a) and widths (b) of the tumors were recorded twice per week, and the tumor volumes were calculated $\left(\mathrm{V}=\mathrm{a} \times \mathrm{b}^{2} / 2\right)$. The mice were sacrificed when the maximal diameter of the tumor exceeded $18 \mathrm{~mm}$ or when the mice seemed moribund, and this day was recorded as the date

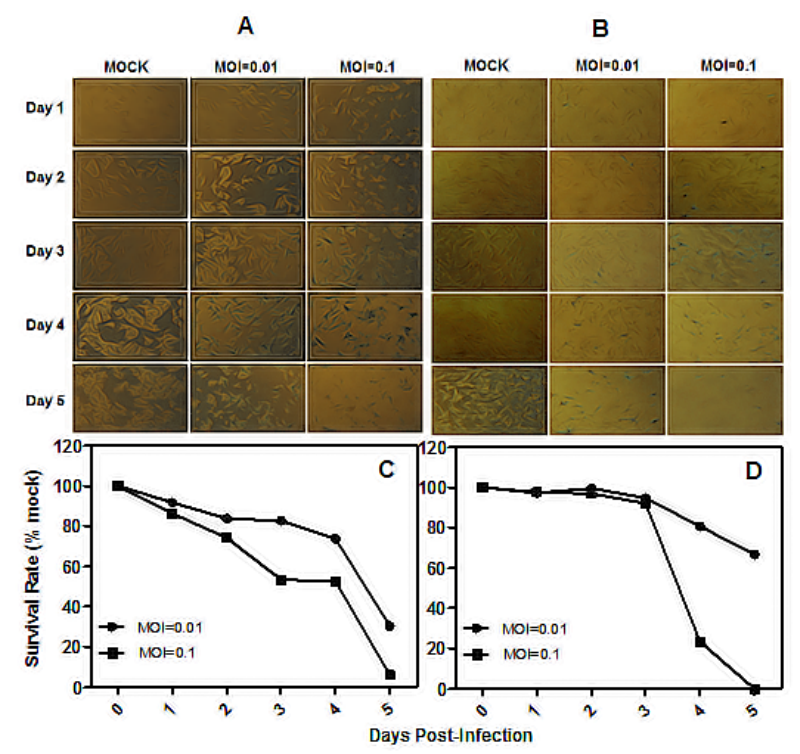

Figure 1. Cytotoxicity of 2 Thyroid Carcinoma Cell Lines in vitro. (A) X-gal staining of ARO cells infected with G47 $\Delta$. Monolayers of ARO cells in 6-well dishes were infected with G47 $\Delta$ or mock virus and incubated with RPMI 1640 medium $/ 1 \%$ heat-inactivated $\mathrm{FBS}$ at $37^{\circ} \mathrm{C}$. X-gal staining was performed each day, and infected cells expressing Lac Z were stained blue. (B) X-gal staining of FRO cells infected with G47 $\triangle$. (C and D) Monolayers of ARO and FRO cells in 6-well dishes were infected with $\mathrm{G} 47 \Delta$ at $\mathrm{MOI}=0.01$ or $\mathrm{MOI}=0.1$ and incubated in RPMI 1640 medium/1\% heat-inactivated FBS at $37^{\circ} \mathrm{C}$, and the number of viable cells was counted each day. The data represent the results of 3 independent trials 


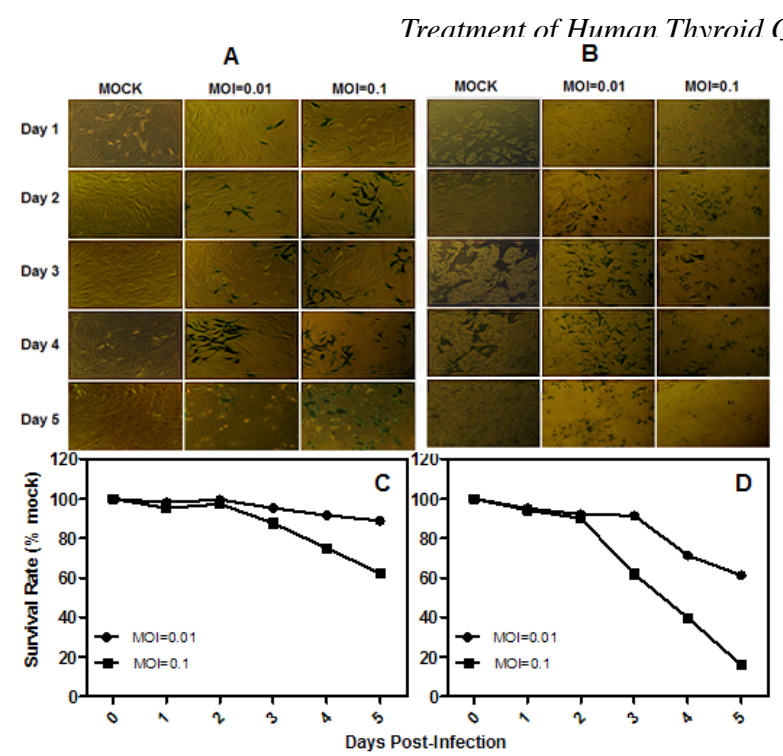

Figure 2. Cytotoxicity of 2 Thyroid Carcinoma Cell Lines in vitro. (A) X-gal staining of WRO cells infected with G47 $\Delta$. Monolayers of WRO cells in 6-well dishes were infected with G47 $\Delta$ or mock virus and incubated with RPMI 1640 medium $/ 1 \%$ heat-inactivated $\mathrm{FBS}$ at $37^{\circ} \mathrm{C}$. X-gal staining was performed each day, and infected cells expressing Lac Z were stained blue. (B) X-gal staining of KAT-5 cells infected with G47 $\Delta$. (C and D) Monolayers of WRO and KAT-5 cells in 6-well dishes were infected with $\mathrm{G} 47 \Delta$ at $\mathrm{MOI}=0.01$ or $\mathrm{MOI}=0.1$ and incubated in RPMI 1640 medium/1\% heat-inactivated FBS at $37^{\circ} \mathrm{C}$, and the number of viable cells was counted each day. The data represent the results of 3 independent trials

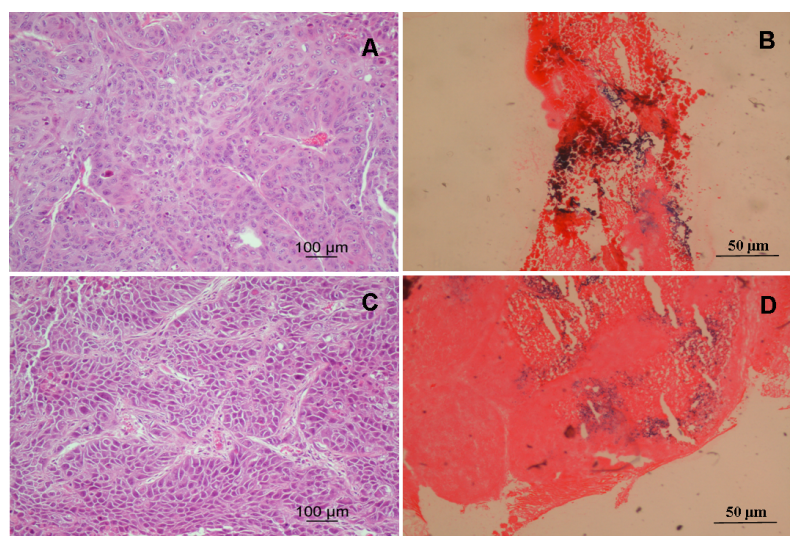

Figure 3. Established s.c. tumors derived from the ATC cell lines ARO $\left(3 \times 10^{6}\right)$ and FRO $\left(3 \times 10^{6}\right)$. ATC cells were suspended in $100 \mu \mathrm{l}$ of RPMI 1640 containing 25\% Matrigel (BD Biosciences) and then s.c. implanted into the left flanks of Balb/c nude mice (4 weeks old). When the s.c. tumors were palpable, $2 \times 107 / 50 \mu \mathrm{l}$ of G47 $\Delta$ or virus buffer $[150 \mathrm{mM} \mathrm{NaCl}$ and $20 \mathrm{mM}$ Tris, (pH 7.5)] was injected into the s.c. tumors twice per week. (A and B) Hematoxylin and eosin staining of ARO and FRO s.c. tumors $(\times 200)$. (C and D) Coronal section through ARO and FRO s.c. tumors of Balb/c nude mice 3 days after G47D inoculation to illustrate viral replication in the s.c. tumors. Coronal sections were stained with $\mathrm{X}$-gal and counterstained with eosin to identify replicating $\mathrm{G} 47 \Delta$ (blue)

of death for the survival studies. The s.c. tumors were excised for hematoxylin-eosin staining. Another 2 nude mice were sacrificed 3 days after G47 $\Delta$ inoculation. The tumors were excised and frozen at $80^{\circ} \mathrm{C}$, cryostat sections $10 \mu \mathrm{m}$ in thickness were prepared, and $\mathrm{X}$-gal staining was performed according to the manufacturer's recommended protocol (Beyotime Institute of Biotechnology, China). Sections were stained with X-gal, and counterstained with eosin to identify cells containing replicating G47 $\Delta$ (blue).

\section{Results}

\section{In vitro cytotoxicity}

To assess the susceptibility of human thyroid carcinoma cell lines to G47 $\Delta$, various thyroid carcinoma cells were infected with G47 $\Delta$ at a low MOI (MOI 0.01 and 0.1 ). For ARO cells, greater than $70 \%$ and $90 \%$ of cells were killed at $\mathrm{MOI}=0.01$ and $\mathrm{MOI}=0.1$, respectively, on day 5 (Figure 1C). For FRO cells, greater than 35\%
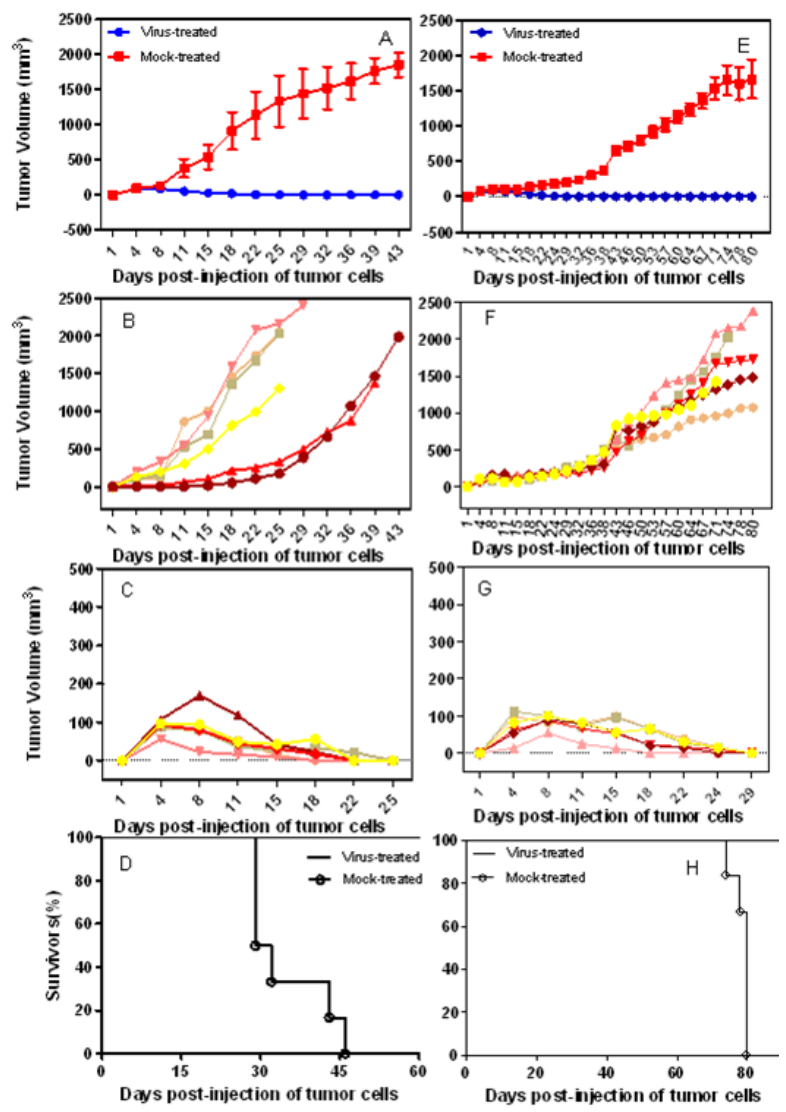

Figure 4. G47 $\Delta$ Significantly Inhibited ATC Growth in vivo and Prolonged Mouse Survival. Tumor volume was recorded twice per week. The mice were sacrificed when the maximal diameter of the tumor exceeded $18 \mathrm{~mm}$ or when the mice seemed moribund, and this day was recorded as the date of death for survival studies. G47 $\Delta$ significantly inhibited tumor growth compared to the control group, and all of the tumors in the treated group regressed completely. (A) The mean tumor volume is shown for the virus-treated and mock-treated ARO groups at different time points. (B and C) The growth of individual treated tumors in the mock- and virus-treated ARO groups, respectively. (D) The median survival time increased from 27 days for the control group to 60 days for the G47 $\Delta$-treated s.c. ARO tumor group $(\mathrm{P}<0.05$, log-rank test). (E) The mean tumor volume is shown for the virus- and mock-treated FRO groups at different time points. ( $\mathrm{F}$ and $\mathrm{G}$ ) Growth of individual treated tumors in the mock- and virus-treated FRO groups, respectively. $(\mathrm{H})$ The median survival time increased from 80 days for the mocktreated group to 90 days for the G47 $\Delta$-treated s.c. FRO tumor group $(p<0.05$, log-rank test $)$ 
of cells were killed at MOI $=0.01$ on day 5 , while $100 \%$ of cells were killed at MOI=0.1 on day 5 (Figure 1D). For WRO cells, $21.1 \%$ and $37.8 \%$ cells were killed at $\mathrm{MOI}=0.01$ and $\mathrm{MOI}=0.1$, respectively, on day 5 (Figure 2C). For KAT-5 cells, greater than $30 \%$ and $80 \%$ cells were killed at $\mathrm{MOI}=0.01$ and $\mathrm{MOI}=0.1$, respectively, on day 5 (Figure 2D). G47 $\Delta$ contains the lacZ gene and can therefore be traced both in vitro and in vivo. Similar to the cytotoxicity results, $\mathrm{X}$-gal staining revealed that $\mathrm{G} 47 \Delta$ effectively replicated and spread among ARO (Figures 1A), FRO (Figures 1B), WRO (Figures 2A) , and KAT-5 cells (Figures 2B).

\section{In vivo study}

ATC is very aggressive and is considered a fatal disease. The majority of patients initially present with a rapidly enlarging tumor in the neck (Are and Shaha, 2006). To determine the therapeutic effects of G47 $\Delta$ on thyroid cancer in vivo, we selected 2 ATC cell lines, ARO and FRO, to form s.c. tumors in Balb/c nude mice, after which we injected G47 $\Delta$ or virus buffer i.t. To evaluate G47 $\Delta$ replication, the virus-treated nude mice were sacrificed 3 days after G47 $\Delta$ injection, and X-gal staining was performed on the sectioned ATC tumors. Blue-stained sections were evaluated for G47 $\Delta$ replication in ARO (Figures 3B) and FRO s.c. tumors (Figures 3D).

Similar to the results obtained in vitro, G47 $\Delta$ elicited prominent antitumor effects and demonstrated a significant inhibition of tumor growth (Figures $4 \mathrm{~A}$ and $\mathrm{E}$ ). In G47 $\Delta$ treated mice bearing s.c. ARO and FRO tumors, all of the tumors completely regressed as a result of treatment (Figures $4 \mathrm{C}$ and $\mathrm{G}$ ). In addition, none of the mice appeared to be moribund, so they were sacrificed on days 60 (ARO) or 90 (FRO). In contrast, the tumors grew rapidly in the control group (Figures $4 \mathrm{~B}$ and $\mathrm{F}$ ). Due to the rapid growth of ARO tumors, all of the mice in the control group were sacrificed by day 47 , with a median survival time of 27 days (Figures 4D). For the FRO tumors, the median survival time was 80 days (Figures $4 \mathrm{H}$ ). G47D significantly prolonged the survival of mice bearing ARO or FRO tumors $(p<0.05, \log$-rank test).

\section{Discussion}

The use of an oncolytic virus is a relatively new therapeutic strategy for cancers. oHSV was first used for the treatment of glioblastoma in 1991 (Martuza et al., 1991). Since then, more than 20 different oHSVs have been used to treat various solid tumors in preclinical studies, including bladder, breast, colorectal, gastric, glioma, head and neck, liver, melanoma, neuroblastoma, pancreatic, and prostate, and ovarian (Reinblatt et al., 2007). Currently, six oncolytic HSV vectors, G207, G47 $\Delta$, 1716, HF10, NV1020, and OncovexGM-CSF (talimogene laherparepvec) have progressed to clinical trials, with OncovexGM-CSF successfully reaching its primary endpoint in a randomized phase III trial for metastatic melanoma (Campadelli-Fiume et al., 2011).

The results of our previous studies indicated that oHSV G47 $\Delta$ was an effective and safe therapy for breast and nasopharyngeal carcinomas (Wang et al., 2011a;
Wang et al., 2011b; Li et al., 2012; Zeng et al., 2013a; Zeng et al., 2013b). G47 $\Delta$ effectively killed breast cancer cells both in vitro and in vivo but spared normal breast epithelial cells (Wang et al., 2011a). G47D is a thirdgeneration oHSV, which was constructed from G207, and contains 3 deletions/mutations that result in selective cytotoxicity to tumor cells (Todo et al., 2001). In addition, both copies of the $\gamma 34.5$ gene are deleted in this vector, which removes the major HSV neurovirulence gene and precludes the shut-off of protein synthesis in response to viral infection in host cells (Mohr et al., 2001); thus, this mutation attenuates neurovirulence, decreases the chance of reverting to wild-type virus, and causes the virus to preferentially replicate in tumor cells. The ICP6 gene encodes the large subunit of ribonucleotide reductase, which is the key enzyme involved in deoxyribonucleic acid synthesis and viral DNA synthesis in nondividing cells (but not in dividing cells) (Goldstein and Weller, 1988). The ICP6 gene is inactivated by insertion of the lacZ gene, which confers tumor cell selectivity to the virus. The $\alpha 47$ gene and the promoter region of the US11 gene are also deleted, which places the US11 gene under the control of the immediate-early $\alpha 47$ promoter. These mutations enhance the viral replication ability and increase the antitumor immune response by increasing MHC class I presentation (Todo et al., 2001).

Although differentiated thyroid cancer is the most common type of thyroid cancer and the majority of patients experience positive outcomes, the 10-year recurrence rate among elderly patients with tumors larger than $4 \mathrm{~cm}$ in diameter or those with tumors that have spread beyond the thyroid, such as those with lymph node metastases, ranges from $20 \%$ to $30 \%$ (Sherman et al., 2008). MTC represents $5 \%$ to $8 \%$ of all thyroid cancers and is more aggressive. One-half of all MTC patients initially develop distant metastases, and the 10-year survival rate is $10 \%$ for these patients. Moreover, no treatment has been proven to prolong the survival of these patients (Pacini et al., 2010). Although ATC represents less than $2 \%$ of all thyroid cancers, it accounts for approximately $40 \%$ of thyroid cancer mortality (Lai et al., 2005). Therefore, new therapies are needed to target thyroid tumors, especially metastases and ATC.

In the present study, we evaluated the cytopathic effects of the third-generation oHSV G47 $\Delta$ on different types of human thyroid carcinoma cells in vitro. G47 $\Delta$ effectively killed multiple types of thyroid tumor cells, with the ATC cell lines ARO and FRO displaying high sensitivity to G47D. The ARO cell line was especially sensitive at low MOI, while the DTC cell lines KAT-5 and WRO showed modest sensitivity. ARO was previously found to be one of the more sensitive thyroid cancer lines to NV1023, another oHSV with different mutations (Yu et al., 2004). KAT-5 was reported to express high levels of nectin-1, one of the HSV receptors, while WRO did not to express nectin-1, which might explain its poor sensitivity here except we found reasonable X-gal staining, indicative of virus infection (Huang et al., 2007). Finally, we found that G47 $\Delta$ effectively inhibited ARO and FRO s.c. tumor growth in vivo. All of the tumors completely regressed in the G47 $\Delta$ treatment group, and survival in this group was 
significantly prolonged. Moreover, no mouse displayed any side effects due to G47 $\Delta$ administration. Yu et al showed that ARO tumors were also sensitive to NV1023, although only 4 of 6 mice remained tumor-free long term after 3 i.t. injections (Yu et al., 2004).

In conclusion, the third-generation oHSV G47A effectively killed different types of human thyroid carcinoma cells in vitro. Of particular interest, G47 $\Delta$ significantly inhibited ATC growth in vivo and prolonged animal survival in 2 different tumors, indicating its potential as an effective treatment for different types of thyroid tumors.

\section{Acknowledgements}

Our work is supported by the National Natural Science Foundation of China (No. 81372815, 81172523).

\section{References}

Are C, Shaha AR (2006). Anaplastic thyroid carcinoma: biology, pathogenesis, prognostic factors, and treatment approaches. Ann Surg Oncol, 13, 453-64.

Campadelli-Fiume G, De Giovanni C, Gatta V, et al (2011). Rethinking herpes simplex virus: the way to oncolytic agents. Rev Med Virol, 21, 213-26.

Goldstein DJ, Weller SK (1988). Factor (s) present in herpes simplex virus type 1-infected cells can compensate for the loss of the large subunit of the viral ribonucleotide reductase: characterization of an ICP6 deletion mutant. Virology, 166, 41-51.

Huang YY, Yu Z, Lin SF, et al (2007). Nectin-1 is a marker of thyroid cancer sensitivity to herpes oncolytic therapy. JClin Endocrinol Metab, 92, 1965-70.

Lai ML, Faa G, Serra S, et al (2005). Rhabdoid tumor of the thyroid gland: a variant of anaplastic carcinoma. Arch Pathol Lab Med, 129, 55-7.

Li J, Zeng W, Huang Y, et al (2012). Treatment of breast cancer stem cells with oncolytic herpes simplex virus. Cancer Gene Ther, 19, 707-14.

Lin SF, Gao SP, Price DL, et al (2008). Synergy of a herpes oncolytic virus and paclitaxel for anaplastic thyroid cancer. Clin Cancer Res, 14, 1519-28.

Martuza RL, Malick A, Markert JM, et al (1991). Experimental therapy of human glioma by means of a genetically engineered virus mutant. Science, 252, 854-6.

Mohr I, Sternberg D, Ward S, et al (2001). A herpes simplex virus type 1 gamma34.5 second-site suppressor mutant that exhibits enhanced growth in cultured glioblastoma cells is severely attenuated in animals. $J$ Virol, 75, 5189-96.

Pacini F, Castagna MG, Cipri C, et al (2010). Medullary thyroid carcinoma. Clin Oncol ( $R$ Coll Radiol), 22, 475-85.

Reinblatt M, Pin RH, Fong Y (2007). Herpes viral oncolysis: a novel cancer therapy. J Am Coll Surg, 205, S69-75.

Russell SJ, Peng KW, Bell JC (2012). Oncolytic virotherapy. Nat Biotechnol, 30, 658-70.

Sherman SI, Wirth LJ, Droz JP, et al (2008). Motesanib diphosphate in progressive differentiated thyroid cancer. $N$ Engl J Med, 359, 31-42.

Sipos JA, Mazzaferri EL (2010). Thyroid cancer epidemiology and prognostic variables. Clin Oncol ( $R$ Coll Radiol), 22, 395-404.

Smallridge RC, Ain KB, Asa SL, et al (2012). American thyroid association guidelines for management of patients with anaplastic thyroid cancer. Thyroid, 22, 1104-39.
Todo T, Martuza RL, Rabkin SD, et al (2001). Oncolytic herpes simplex virus vector with enhanced MHC class I presentation and tumor cell killing. Proc Natl Acad Sci USA, 98, 6396-401.

Vanderpump MP (2011). The epidemiology of thyroid disease. Br Med Bull, 99, 39-51.

Varghese S, Rabkin SD (2002). Oncolytic herpes simplex virus vectors for cancer virotherapy. Cancer Gene Ther, 9, 967-78.

Wang J, Hu P, Zeng M, et al (2011a). Oncolytic herpes simplex virus treatment of metastatic breast cancer. Int J Oncol, 40, 757-63.

Wang JN, Hu P, Zeng MS, et al (2011b). Anti-tumor effect of oncolytic herpes simplex virus G47delta on human nasopharyngeal carcinoma. Chin J Cancer, 30, 831-41.

Yu Z, Eisenberg DP, Singh B, et al (2004). Treatment of aggressive thyroid cancer with an oncolytic herpes virus. Int J Cancer, 112, 525-32.

Zaman M, Fatima N, Sajjad Z, et al (2012). Threshold primary tumour sizes for nodal and distant metastases in papillary and follicular thyroid cancers. Asian Pac J Cancer Prev, 13, 2473-6.

Zeng W,Hu P, Wu J, et al (2013a). The oncolytic herpes simplex virus vector G47 effectively targets breast cancer stem cells. Oncol Rep, 29, 1108-14.

Zeng WG, Li JJ, Hu P, et al (2013b). An oncolytic herpes simplex virus vector, G47Delta, synergizes with paclitaxel in the treatment of breast cancer. Oncol Rep, 29, 2355-61. 